\title{
OSA: An awaking disorder!
}

\author{
Srihari R. ${ }^{1}$, Mathangi D.C. ${ }^{2}$, Shyamala R. ${ }^{3}$ \\ 1, 2, 3, (Department of Physiology, Chettinad Hospital \& Research Institute, Kelambakkam, Tamil Nadu 603 103, India,)
}

\begin{abstract}
OSA is characterized by repeated narrowing or collapse of the upper airway during sleep. It is the most common sleep disordered breathing seen among general population and contributes to increased morbidity and mortality. Recent lifestyle modifications have made Indian population more prone to this chronic disorder. Epidemiological studies show an increase in OSA prevalence among Indian population. OSA is associated with various co-morbid conditions which can be easily prevented by early diagnosis for which awareness should be brought among practicing Indian physicians and general population.
\end{abstract}

Keywords- CPAP, co-morbidities, obstructive sleep apnea, polysomnography (PSG), risk factors

\section{INTRODUCTION}

Recent lifestyle modifications due to rapid growth in science and technology have led to various changes in sleeping pattern of human, which is an essential component of life. Disturbance in sleep pattern hinders various physiological functions initially and subsequently leads to lifestyle disorder. Sleep disordered breathing (SDB), a lifestyle disorder is characterized by frequent ventilatory overshoots and undershoots with swings in arterial blood gas levels [1]. One such type of sleep disordered breathing is sleep apnea. Apnea is a condition in which absence of spontaneous breathing occurs more than 10seconds with decrease in airflow $>90 \%$ and hypopnea is decrease in airflow $\geq 30 \%$ more than 10seconds [2]. Sleep apnea is a condition in which apnea occurs frequently during sleep causing disturbance in body homoeostasis. Generally, sleep apnea (a SDB) is divided into central, obstructive and mixed. Central sleep apnea(CSA) is due to suppression of central respiratory centers leading to reduced or cessation of motor output to pharyngeal muscles causing collapse of the airway [1].

Obstructive sleep apnea (OSA) or pickwickian disease is a potentially disabling condition characterized by repeated narrowing or collapse of the upper airway during sleep. Episodes of complete or partial airway narrowing are often associated with oxygen desaturation and terminated by an arousal from the sleep [3]. Mixed type is a combination of central and obstructive sleep apnea and seen in very few patients. OSA is the most common SDB seen in the general population and recognized as increased cause of morbidity and mortality [4]. Severity of OSA is diagnosed using Apnea-Hypopnea index (AHI). AHI is defined as the number of apneas and hypopneas per hour of sleep. Mild OSA - AHI between 5-15, moderate OSA - AHI between 15-30 and severe OSA - AHI >30 per hour of sleep. Obstructive sleep apnea syndrome (OSAS) is clinically characterized by AHI $>5$ with excessive daytime sleepiness (EDS). Snoring is recognized as a characteristic feature of OSA [5], but, all snorers are not diagnosed with OSA. Snoring in absence of OSA is diagnosed when habitual snoring occurs with AHI <5 episodes per hour of sleep and absence of daytime symptoms.

Various unidentified forms of sleep apnea were reported in $19^{\text {th }}$ century itself. In 1870's British physicians described OSA as 'fruitless contraction of inspiratory and expiratory muscles against closed glottic obstruction with accompanying cyanosis during sleep' [6]. Finally, Gastaut et al [7] recognised OSA in obese cases in 1960's, following which physiological research in sleep and breathing exploded in a huge manner. From 1990's, there is an increase in physiological research and clinical approach towards this chronic debilitating clinical disorder.

\subsection{Classification}

OSA is due to obstruction of the upper airway that leads to apneic episodes during sleep. Fujita et al [8] had classified the anatomical obstruction levels of OSA as follows:

Type 1: collapse in retropalatal region only

Type 2: collapse in retropalatal and retrolingual region

Type 3: collapse in retrolingual region only.

Retropalatal region is seen as the most common site of collapse [1] 


\subsection{Prevalence of OSA}

Worldwide, OSA has been recognized as a major contributor to morbidity and mortality [4]. OSA prevalence is not the same among various ethnicities. Epidemiological surveys say that prevalence of OSA worldwide is $4 \%$ in males and $2 \%$ in females [9]. The prevalence is found to be more in developed and in developing countries. India being a fast developing country has prevalence of OSA also rising. A study conducted in semi-urban Delhi population suggested OSA prevalence to be $19.7 \%$ in men and $7.4 \%$ in women [4]. Another study conducted in northern India revealed an overall prevalence of $9.3 \%$ for OSA with $13.5 \%$ in male and 5.5\% in female [10]. These studies suggest that OSA is undoubtedly increasing in India

\section{RISK FACTORS OF OSA}

OSA is a multifactorial disorder. Various risk factors include increasing age[4,5], male gender[4,6,11], snoring[5,12,13], habitual smoking[4], alcohol intake[4,5], obesity[4,5,12], high waist-hip ratio(WHR)[5,13], increased Neck circumference(NC)[11,13,14,15], percent predicted neck circumference (PPNC) - a height corrected neck measure of neck circumference which is calculated using formula PPNC $=\left(1000^{*} \mathrm{NC}\right.$ in $\mathrm{cm}) /(0.55 *$ height in $\mathrm{cm}+310)[16]>90$

Epidemiological studies shows that prevalence of OSA in higher in men than women $[9,10,11]$. The exact mechanism by which the male predominance exists is still unclear. But, various anatomical and physiological factors have been suggested to support this male predominance. In general males have increased NC, pharyngeal airway length and volume and increased soft palate volume than females which can cause OSA $[17,18]$. Though, men have larger pharynx than women there exists a vast change in pharyngeal area with lungvolume change than women which predisposes to airway collapse [19]. Pharyngeal airway in men was found to be more collapsible during sleep than women [20]. Testosterone, male sex hormone also increases upper airway collapsibility during sleep and induce/aggravate OSA in men [21,22,23].

Obesity is considered as an important risk factor of OSA [12,24]. In India, obese individuals have four times higher risk of developing OSA than non-obese individuals $[4,10]$. Obesity predisposes an individual to OSA by increasing fat deposits in the body leading to increase in WHR and NC. Increased adiposity in the neck directly mass loads the airway and predisposes to collapse, hence OSA [25]. But, 50\% of the OSA patients were reported to be non-obese too [24]. The proposed reason is that in non-obese male OSA patients muscle mass content is almost less but the neck fat content remains the same. In addition, fat content in male neck is proportionately higher than other parts of the body which may also influence OSA preponderance [26].

\section{CONSEQUENCES OF OSA}

Studies on OSA patients have shown that it is associated with co-morbid conditions like cognitive, metabolic and cardiovascular consequences and affects the quality of life.

\subsection{Cognitive consequences}

OSA as it is characterized by intermittent hypoxia results in decrease in grey matter and network integrity in brain lobes causing impairment of vigilance, memory, psycho-motor performance, attention etc., [1]. Sleep fragmentation, a characteristic feature in OSA induced by frequent apnea causes dysfunction of neural networks in frontal lobe thus disturbing memory and cognitive functions [27].

\subsection{Metabolic consequences}

OSA by various mechanisms results in metabolic disturbances which ultimately disturbs the body homeostasis. OSA patients tend to be more prone to Type 2 Diabetes Mellitus. The probable mechanism could be that chronic intermittent hypoxia leads to high sympathetic activity which increases insulin resistance and decreased glucose tolerance and predisposes to DM in untreated patients of OSA [1,28].

\subsection{Cardiovascular consequences}

OSA patients have been reported to have increased risk of cardio-vascular diseases, OSA patients were found to have high hypertension. The probable mechanism is that OSA due to intermittent hypoxia leads to excessive daytime sympathetic vasoconstrictor activity, increased free radicals or reactive oxygen species, elevated levels of pro-inflammatory mediators such as C-reactive protein, TNF- $\alpha$, IL- $1 \beta$, IL-6 and adhesion molecules which causes local and systemic inflammation promoting CAD, pulmonary hypertension and even Left ventricular failure $[1,29]$.

OSA patients are prone to have metabolic syndrome, which is a combination of type-2 DM, Hypertension and dyslipidaemia. A combination of metabolic syndrome and OSA has been classified as Syndrome Z [30]. Treating OSA patients with CPAP (Continuous Positive Airway Pressure), the most effective treatment for OSA, decreases lipid profile [29,30]. 


\subsection{Decreased quality of life}

Lack of nocturnal sleep, a main feature in OSA patients, results in increased day time somnolence. It leads to decreased memory, attention, work performance, nervousness, impaired sexual performance [13], increased vehicular accidents [31], psychological depression [32] and decreased immune and general health which can affect physical and mental well being of an individual.

\section{DIAGNOSIS}

OSA initially manifests as snoring [12], if untreated subsequently progresses to OSA. OSA patients exhibit variety of symptoms. Nocturnal symptoms include loud snoring [4,13], frequent apneas commonly witnessed by bed partner, gasping and choking leading to arousals, and restless sleep. Daytime symptoms include EDS (Excessive daytime sleepiness), morning headache, sore throat, decreased memory and depression [13]. EDS is assessed using Epworth Sleepiness scale. A score $>10$ is considered sleepy. There are other questionnaires also like Berlin questionnaire, STOP questionnaire, etc.,

A complete physical body examination including BMI, waist hip ratio, neck circumference and blood pressure gives data related to obesity and OSA. Oropharyngeal patency is assessed using modified Mallampati score which grades the positions accordingly.

I - Entire uvula seen with tongue at rest

II - Partial view of uvula seen

III - Soft and hard palate seen

IV - Only hard palate seen

Polysomnography (PSG) is the 'gold standard' method to diagnose OSA. It records various parameters related to sleep and helps in diagnosing OSA. It is performed as a single night sleep study either in a sleep lab or in home under supervision of a sleep technician. It records electroencephalogram (brain activity), electrocardiogram (heart activity), electooculogram (eye movements),submental and tibialelectromyogram (muscle activity), respiratory movements, nasal airflow and oxygen saturation level. Apneic episodes can be easily recorded using PSG studies.

\section{TREATMENT}

Treatment includes medical and surgical management. Medical management includes weight loss, Continuous positive airway pressure (CPAP) and oral appliances. Obesity is directly related to OSA [12]. Hence weight loss decreases fat deposition in the body especially in the neck, chest and abdomen, which prevents pharyngeal collapse and increases tracheal traction and lung volume. CPAP is the 'gold standard' medical management for OSA. Application of CPAP acts as a pneumatic splint maintains positive pressure at pharyngeal level and prevents collapse. CPAP usage has also reported to reduce OSA related co-morbid condition [33]. Application of oral appliances may also be used in some patients.

Surgical techniques include Uvulopalatopharyngoplasty(UPPP) the most common surgical procedure for treating OSA, in which palatal obstruction is eliminated by resecting redundant palatal and pharyngeal tissues [34]. Other procedures include lingualplasty, glossectomy and radiofrequency tongue ablation, genioglossal advancement and hyoid myotomy.

\subsection{Hypoglossal-nerve stimulation}

Reduced upper airway muscle activity leads to OSA. Hypoglossal nerve innervates genioglossus the important upper airway dilator. Hence stimulation of hypoglossal nerve which is usually done using Transcutaneous electrical nerve stimulator (TENS), causes contraction of genioglossus leading to airway opening and preventing its collapse.

\section{CONCLUSION}

Lifestyle modifications have resulted in an increase in prevalence of OSA among Indian population. OSA has been associated with various co-morbid conditions which if goes unnoticed shall become detrimental to the individual. Awareness among Indian practicing physicians on SDB was only 10\% [35] which should be rectified by updating sleep medicine among practicing physicians to identify, diagnose and treat patients with the awaking disorder - OSA.

\section{ACKNOWLEDGEMENTS}

I would like to thank Dr. Prema.Sembulingam, Dr. K.Sembulingam and Dr. B.Viswanath Rao of Madha Medical College and Research Institute for their valuable guidance and support in bringing out this publication. My thanks are due to Mrs.S.Nasreen for her technical help. 


\section{REFERENCES}

[1] JA. Dempsey, SC. Veasey, BJ. Morgan, and CP.O’Donell, Pathophysiology of Sleep Apnea, Physiol Rev, 90, $2012,47-112$.

[2] C. Iber, S. Ancoli-Israel, A.Chesson, and S.Quan, AASM Manual for the scoring of sleep Associated Events:Rules, Terminology and Technical Specifications. $1^{\text {st }}$ ed. Westchester, illiois: American Academy of Sleep Medicine, 2007

[3] American Academy of Sleep Medicine Task Force. Sleep-related breathing disorders in adults:recommendations for syndrome definition and measurement techniques in clinical research. The Report of an American Academy of Sleep Medicine Task Force, Sleep,22, 1999, 667-689.

[4] SK. Sharma, S.Kumpawat, Banga A, and Goel A, Prevalence and risk factors of obstructive sleep apnea syndrome in a population of Delhi, India, Chest,130(1), 2006, 149-156

[5] PC Deegan, WT. McNicholas, Predictive values of clinical features for the obstructive sleep apnoea syndrome, Eur Respir J, 9 , 1996, 117-124.

[6] P Lavie, Restless nights: Understanding snoring and sleep apnea, New Haven, CT: Yale Univ. Press 2003.

[7] H. Gastaut, CA Tassinari, B and Duron B, Polygraphic study of the episodic diurnal and nocturnal (hypnic and respiratory) manifestations of the pickwick syndrome, Brain Res, 1, 1966, 167-186.

[8] S. Fujita, Pharyngeal surgery for obstructive sleep apnoea and snoring, in Fairbanks DNF (Ed.), Snoring and obstructive sleep apnoeas, (New York: Raven Press;1987), 101-128.

[9] T. Young, M. Palta, J, Dempsey, et al, The occurrence of sleep disordered breathing among middle-aged adults, $N$ Engl $J$ Med, 328, 1993, 1230-1235.

[10] EV Reddy, T. Kadhiravan, HK Mishra, V Sreenivas, KK Handa, S Sinha, and SK Sharma, Prevalence and risk factors of obstructive sleep apnea among middle-aged urban indians: A community based study, Sleep Medicine, 10(8), 2009, 913-918.

[11] SM Banabilh, AR Samsuddin, AH Suzina, and S Dinsuhaimi, Facial profile shape, Malocclusion and Palatal Morphology in Malay Obstructive Sleep Apnea Patients, Angle Orthodontist, 80(1), 201037-42.

[12] IE Gabbay.,P Lavie P, Age and gender related characteristics of OSA, Sleep breath,16(2), 2012, 453-460.

[13] F Hora, LM Napolis, C Daltro, SK Kodaira, S Tufik, SM Togeiro ,LE and Nery LE, Clinical, anatomical and upper-airway characteristics of obese patients with obstructive sleep apnoea syndrome, Respiration,74(5), 2007, 517-524.

[14] JA Pinto, LB Mello Godoy, and VW Pinto Brandao Marquis, Anthropometric data as predictors of Obstructive Sleep Apnea severity, Braz J Otorhinolaryngol,77(4), 2011, 516-521.

[15] M Karimi, J Koranyi, Franco C et al, Increased neck soft tissue mass and worsening of obstructive sleep apnea after growth hormone treatment in men with abdominal obesity, Journal of Clinical Sleep Medicine, 6(3), 2010, 256-263.

[16] RJ O Davies, JR Stradling, The relationship between neck circumference, radiographic pharyngeal anatomy and obstructive sleep apnoea, EurRespir J,3, 1990, 504-509.

[17] A Onat, G Hergenç, H Yüksel et al, Neck circumference as a measure of central obesity : Associations with metabolic syndrome and obstructive sleep apnea syndrome beyond waist circumference, Clinical Nutrition, 28(1), 2009, 46-51.

[18] A. Malhotra, Y. Huang, RB Fogel et al, The male predisposition to pharyngeal collapse: importance to airway length, American Journal of Respiratory Critical Medicine, 166(10), 2012, 1388-1395.

[19] LJ Brooks, KP Strohl, Size and mechanical properties of the pharynx of healthy man and women, Am Rev Respir Dis, 146(6), 1992, 1394-1397.

[20] A. Pillar, A. Malhotra A, RB Fogel et al, Airway mechanics and ventilation in response to resistive loading during sleep. Influence of gender, Am J Resp Crit Care Med,162, 2000, 1627-1632.

[21] PA Cistulli,RR Grunstein, CE and Sullivan CE, Effect of testosterone on upper airway collapsibility during sleep, Am J Respir Crit Care Med,149, 1994, 530-532.

[22] PY Liu, B Yee, SM Wishart SM et al, The short term effects of high dose testosterone on sleep, breathing and function in older men, Journal Clinical Endocrinology Metabolism, 88(8), 2003, 3605-3613.

[23] RE Sandblom, AM Matsumoto, and RB Schoene, Obstructive Sleep Apnea Syndrome Induced by Testosterone Administration, N Engl J Med,308, 1983508-510.

[24] IL Mortimore , I Marshall, PK Wraith et al, Neck and total body fat deposition in nonobese and obese patients with sleep apnea compared with that in control subjects, American Journal of Respiratory Critical Care Medicine,157(1), 1998, $280-283$.

[25] KE Shelton, H Woodson, S Gay et al, Pharyngeal fat in obstructive sleep apnea, Am. Rev. Respir. Dis, 148, 1993, 462-466

[26] AT Whittle, I Marshall, IL Montimore et al, Neck soft tissue and fat distribution:comparison between normal men and women by magnetic resonance imaging, Thorax, 54, 1999, 323-328.

[27] E Verstraeten , R Cluydts, Executive control of attention in sleep apnea patients: theoretical concepts and methodological considerations, Sleep Med Rev, 8, 2004, 257-267.

[28] JE Dimsdale, T Coy, MG Ziegler et al, The effect of sleep apnea on plasma and urinary catecholamines, Sleep, 18, 1995, 377381.

[29] U Haptipogulu, I Rubinstein, Inflammation and obstructive sleep apnea syndrome pathogenesis: A working hypothesis, Respiration, 70(6), 2003, 665-671.

[30] SK Sharma, G Ahulwalia, Epidemiology of adult obstructive sleep apnoea syndrome in India, Indian Journal of Medical Research, 131(2), 2010,171-175.

[31] CF George, Sleepiness, sleep apnoea, and driving: still miles to go before we safely sleep, Am J Respir Crit Care Med,170, 2004, 927-928.

[32] R Sampaio, MG Pereira, and JC Winck, Psychological morbidity, illness representations, and quality of life in female and male patients with obstructive sleep apnea syndrome, Psychology, Health and Medicine, 2011, 1-14.

[33] K Sharma, Prevalence of metabolic syndrome in obstructive sleep apnoea and effect of treatment with auto-continuous positive airway pressure (CPAP) on metabolic syndrome. ClinicalTrials.gov registration number: NCT00694616.http://clinicaltrials.gov/ct2/results?term=NCT00694616.

[34] S Fujita, W Conway ,F Zorick et al, Surgical correction of anatomic abnormalities in obstructive sleep apnoea syndrome:uvulopalatopharyngoplasty, Otolaryngol Head Neck Surg, 89(6), 1981, 923-934.

[35] Ajit Vigg, Avanti Vigg, and Arul Vigg, Awareness of Issues Related to Sleep Disordered Breathing Amongst Practicing Physicians, Indian Journal Chest Disorders Allied sciences, 47, 2005, $25-29$. 